\title{
Kommentierung in gedruckten und digitalen Briefausgaben
}

Seit 1. Januar 2017 läuft das auf acht Jahre angelegte, vom Schweizerischen Nationalfonds und der Jeremias Gotthelf-Stiftung finanzierte Projekt der kommentierten Edition von Jeremias Gotthelfs Korrespondenzen. Die nicht ausschließlich digitale Edition orientiert sich hinsichtlich der Kommentierung an den Standards der Historisch-kritischen Gesamtausgabe der Werke und Briefe von Jeremias Gotthelf sowie überhaupt vieler gedruckter (Brief-)Editionen. Doch wie wird gegenwärtig in digitalen Briefeditionen erläutert? Wo liegen die Gemeinsamkeiten, wo die Unterschiede von Erläuterungen in gedruckten und digitalen Briefeditionen? Diesen Fragen soll im vorliegenden Beitrag nachgegangen werden, wobei von besonderem Interesse ist, wie die verschiedenen Editionen ihre Zielsetzungen begründen und ihr Vorgehen legitimieren.

\section{Die Edition der Korrespondenzen von Albert Bitzius (Jeremias Gotthelf)}

Im Modul Korrespondenz sollen alle überlieferten und der Forschungsstelle Jeremias Gotthelf zugänglichen Schreiben von respektive an Albert Bitzius ediert werden. Unter den schätzungsweise weit mehr als 3000 Dokumenten befinden sich Korrespondenzen mit Freunden und Verwandten, Zeugnisse der Briefwechsel mit schweizerischen und deutschen Verlegern und zahlreiche amtliche Korrespondenzen, die Bitzius als Pfarrer, Aktuar und erster Beisitzer des örtlichen Sittengerichts, als Präsident und später Sekretär der Schulkommission oder als Schulkommissär führte. Zu Beginn des Jahres 2019 waren insgesamt rund 2600 Schreiben von und an Albert Bitzius bekannt, von denen die Mehrheit in der Burgerbibliothek Bern, der Verwalterin großer Teile seines Nachlasses, und im Staatsarchiv des Kantons Bern liegt. Weitere Schreiben sind wie so häufig bei Briefkorpora verstreut und werden in schweizerischen Archiven, europäischen Institutionen und in Übersee aufbewahrt.

Etwa die Hälfte dieser Schreiben ist bislang noch nicht ediert worden, hauptsächlich da sich die Herausgeber der Sämtlichen Werke (1911-1977) in den Briefbänden der Ergänzungsausgabe aus Platzgründen ,,auf das für die Kenntnis der Persönlichkeit und des Werks Gotthelfs Wichtige“ beschränken mussten. ${ }^{1}$ Ausgeklammert wurden

\footnotetext{
Jeremias Gotthelf (Albert Bitzius): Vierter Ergänzungsband. Briefe. Erster Teil. Bearb. von Kurt Guggisberg und Werner Juker. Erlenbach-Zürich 1948 (Sämtliche Werke in 24 Bänden), S. 7. - Zur heutigen Maßstäben nicht mehr genügenden Edition von Gotthelfs Korrespondenzen in den Sämtlichen Werken vgl. Hellmut Thomke: Probleme der Edition und Kommentierung von Gotthelfs Briefwechsel. Der Briefbestand und die unbefriedigenden Kriterien der Auswahl, Wiedergabe und Kommentierung in den vorliegenden Editionen. In: Jeremias Gotthelf - Wege zu einer neuen Ausgabe. Hrsg. von Barbara Mahlmann-Bauer und Christian von Zimmermann. Tübingen 2006 (Beihefte zu editio. 24), S. 115-122.
} 
etwa Kondolenz- oder Gratulationsschreiben und Schreiben, in denen die Herausgeber haltlose und unqualifizierte Beschuldigungen orteten. ${ }^{2}$ Auch aus Rücksichtnahme auf Verwandte und Bekannte blieben manche Schreiben unediert und vermeintlich problematische Passagen wurden geändert oder gestrichen. ${ }^{3}$ Ebenso taxierten die Herausgeber der Ergänzungsbände die Briefe der in der französischsprachigen Schweiz weilenden Tochter Cécile als uninteressantes „Geplauder aus dem Welschland, womit sie dem Vater Freude machen“ wollte. ${ }^{4}$ Auch diese Äußerung ist einem Forschungsverständnis geschuldet, das auf den Autor und die Werkgeschichte verengt ist. Für andere Fragen hingegen könnten Céciles Briefe aufschlussreich sein. So informiert sie über ihr Leben im Welschland, beschreibt den Daheimgebliebenen das von der Uhrenfabrikation geprägte neuenburgische Dorf Dombresson ${ }^{5}$ und vergleicht die Pfarrhäuser mit denjenigen im heimischen Lützelflüh. ${ }^{6}$ Indem die projektierte Edition die irgend ermittelten Schreiben von respektive an Bitzius in ihrer Ganzheit präsentiert, wirkt sie einer verkürzenden Sicht entgegen und eröffnet über das Interesse an Gotthelf hinaus unterschiedliche Zugänge zu diesem Quellenkorpus. Hier ist beispielsweise an die Verwaltungsgeschichte oder an Fragen zu Handlungsfeldern von Pfarrern in liberal regierten Gemeinwesen zu denken; auch politische und Kommunikationsnetzwerke werden in den Korrespondenzen greifbar.

Das Publikationskonzept des auf acht Jahre angelegten Projekts sieht ein mehrstufiges Verfahren vor. Erste Konvolute sollen mit der Aufschaltung der digitalen Gesamtedition $(d H K G)$ digital und im Druck publiziert werden; die Publikation weiterer Korrespondenzbestände erfolgt schrittweise. Während in der kommentierten Bibliotheksausgabe ausgewählte Briefwechsel vorgelegt werden sollen, stellt die $d H K G$ alle Korrespondenzen zur Verfügung. Mit der 2012/13 in Zusammenarbeit mit Telota (Berlin-Brandenburgische Akademie der Wissenschaften) eingeleiteten digitalen Reform, mit der Texte und Kommentare gemäß den Richtlinien der Text Encoding Initiative (TEI) erfasst werden sollen, sind die Voraussetzungen für die digitale Edition geschaffen worden. Aus dem gleichen Datenmaterial können künftig neben der Bibliotheks- eine digitale Ausgabe sowie Popularausgaben erzeugt werden, die unterschiedliche Nutzungsszenarien vorsehen. ${ }^{7}$ Die $d H K G$ soll sich an ein heterogenes Publikum richten, zu dem

\footnotetext{
Jeremias Gotthelf (Albert Bitzius): Achtzehnter Ergänzungsband. Nachträge - Register. Bearb. von Werner und Bee Juker. Erlenbach-Zürich 1977 (Sämtliche Werke in 24 Bänden), S. 336.

3 Siehe: Briefwechsel zwischen Jeremias Gotthelf und Abraham Emanuel Fröhlich. Hrsg. von Rudolf Hunziker. Winterthur 1906, S. 130. Hunzikers Edition bildet die Textgrundlage der Ergänzungsbände. - Vgl. zu Eingriffen von Bitzius’ Tochter in die Sämtlichen Werke Franzisca Pilgram-Frühauf: 100 Jahre Sämtliche Werke von Jeremias Gotthelf. Familienausgabe oder historisch-kritische Edition? In: Berner Zeitschrift für Geschichte 73, 2011, H. 2, S. 3-31.

4 Gotthelf 1977 (Anm. 2), S. 311f.

5 Vgl. BBB (Burgerbibliothek Bern) N Jeremias Gotthelf 25.9.13.4, undatierter Brief (vermutlich vom 11./12. August 1853) von Cécile an Albert Bitzius.

6 Vgl. BBB N Jeremias Gotthelf 25.9.13.2, undatierter Brief (vermutlich vom 7. August 1853) von Cécile an Albert Bitzius.

7 Vgl. etwa Holger Helbig: Person unter Papieren. Über die Folgen digitalen Edierens für die Kategorien Autor und Werk am Beispiel der Uwe Johnson-Werkausgabe. In: Textgenese und digitales Edieren. Wolfgang Koeppens Jugend im Kontext der Editionsphilologie. Hrsg. von Katharina Krüger, Elisabetta Mengaldo und Eckhard Schumacher. Berlin u. a. 2016 (Beihefte zu editio. 40), S. 107-130, hier S. 110, oder Patrick Sahle: Kap. 17. Digitale Edition. In: Digital Humanities. Eine Einführung. Hrsg. von Fotis Jannidis, Hubertus Kohle und Malte Rehbein. Stuttgart 2017, S. 243.
} 
Wissenschaftler verschiedener Disziplinen, interessierte Laien oder auch Schulklassen zählen. Die digitale Edition muss folglich nutzungsspezifische Pfade bereitstellen, weshalb eine Partnerschaft mit der Hochschule der Künste Bern eingegangen worden ist. Eine Forschungsgruppe aus dem Bereich der Signaletik eruiert in enger Zusammenarbeit mit der Forschungsstelle Jeremias Gotthelf in einem methodengeleiteten Verfahren einzelne Nutzungsgruppen und deren Bedürfnisse. ${ }^{8}$ Die technische Umsetzung soll in Zusammenarbeit mit der Nationalen Infrastruktur Editionen ${ }^{9}$ erfolgen, wodurch auch die Langzeitspeicherung gewährleistet würde.

\section{Warum eine digitale Edition?}

Schnell lassen sich etliche Vorteile digitalen Edierens aufzählen. Ihr prinzipiell offener, stets aktualisierbarer Charakter ist vielleicht der gewichtigste Vorzug digitaler Editionen im Allgemeinen und der digitalen Präsentationsform gegenüber einer Buchausgabe im Besonderen. ${ }^{10}$ Gerade bei Korrespondenzen ist damit zu rechnen, dass einzelne Schriftstücke erst zu einem späteren Zeitpunkt aufgefunden oder zugänglich werden. In der digitalen Edition können diese auch nachträglich aufgenommen werden, während ein entsprechender Druckband schon auf dem Ladentisch liegt.

Nicht nur die Textedition, sondern auch der Kommentar, da er bei Vorliegen neuer Erkenntnisse stets erweiter- und korrigierbar ist, könnte von dieser Eigenschaft profitieren. ${ }^{11}$ Ohnehin ist der Nutzen der digitalen Editorik generell unbestritten. Hierzu zählen die Sicherung der technischen Anschlussfähigkeit und die durch die Verwendung von Normdateien gewährleistete Vernetzbarkeit mit weiteren digitalen Ressourcen. So können Metadaten, bei Briefen etwa die Namen der Korrespondierenden, Schreibort und -zeit von Metasuchmaschinen und Suchportalen wie Kalliope ${ }^{12}$ und correspSearch $^{13}$ abgerufen werden, was den Forschenden eine editionsübergreifende Suche ermöglicht. ${ }^{14}$ Die in der Edition ausgezeichneten Personen, Orte und Werke, die als Entitäten mit einem zentralen Register verknüpft sind, bilden die Basis für mehrfach kombinierbare Such- und Filterfunktionen, die dem Nutzer und der Nutzerin der

8 Das wachsende Bewusstsein für die Bedeutung des interface in der digitalen Editorik wird durch die Durchführung von Tagungen bezeugt, etwa an der Universität Graz die Tagung „Digital Editions as Interface“ (23./24. September 2016). Zur Notwendigkeit, Akteure mit verschiedener fachlicher Spezialisierung für die Erarbeitung digitaler Editionen beizuziehen, vgl. Patrick Sahle: Digitale Editionsformen. Zum Umgang mit der Überlieferung unter den Bedingungen des Medienwandels. 3 Teile. Norderstedt 2013 (Schriften des Instituts für Dokumentologie und Editorik. 8), S. 227-242, bes. S. 234. Sahle beschreibt ferner die unterschiedlichen Arbeitsbereiche in Editionsprojekten mit Hilfe des Model View Controller-Paradigmas, vgl. Sahle 2017 (Anm. 7), S. 234-252, hier S. 241-244.

9 https://www.nie-ine.ch/ - Alle in diesem Beitrag zitierten online zugänglichen Ressourcen wurden am 13. Juli 2019 zuletzt geprüft.

10 Siehe etwa Sahle 2013 (Anm. 8), 2, S. 218-226 und S. 238-240.

11 Ulrike Leuschner: Vom Brief zum Werk. Kongruenzen und Divergenzen der Präsentation und Kommentierung am Beispiel der Merck-Edition. In: Brief-Edition im digitalen Zeitalter. Hrsg. von Anne Bohnenkamp und Elke Richter. Berlin u. a. 2013 (Beihefte zu editio. 34), S. 7-23, hier S. 13f.

12 http://kalliope.staatsbibliothek-berlin.de/de/index.html/.

13 http://correspsearch.net/index.xql/.

14 Siehe z. B. Peter Stadler: Interoperabilität von digitalen Briefeditionen. In: Fontanes Briefe ediert. Internationale wissenschaftliche Tagung des Theodor-Fontane-Archivs Potsdam, 18. bis 20. September 2013. Hrsg. von Hanna Delf von Wolzogen und Rainer Falk. Würzburg 2014, S. 278-287. 
digitalen Edition einen ,analysierenden Zugriff“ auf das Material bieten. ${ }^{15}$ Auf digitalen Benutzeroberflächen erleichtern Schaltflächen die Navigation, indem vorangehende oder nachfolgende Briefe des Gesamtkorpus und des Einzelbriefwechsels anwählbar sind. In ansprechenden Visualisierungen können schließlich dank präziser Codierung erste Auswertungen des Materials, etwa als Kalender, Schlagwortwolken oder Korrespondierendennetzwerke, ${ }^{16}$ bereitgestellt werden. Insgesamt ermöglicht digitales Edieren dank des transmedialen Charakters der Daten, also der strikten Trennung von Inhalt und Form, eine variable Präsentation der Editionsinhalte (single-source-Prinzip) und somit verschiedene Sichten auf das Dokument resp. den Text. ${ }^{17}$

Die digitale Editions- und Präsentationsweise bietet unter der Voraussetzung präziser Codierung also bedeutend mehr Funktionalitäten und Anschlussmöglichkeiten als eine gedruckte Publikation. Neben vereinzelten kritischen Stimmen, etwa zur Verlinkung oder zur Hoffnung auf eine Beschleunigung von Erkenntnisprozessen durch die maschinelle Auswertbarkeit von Daten, ${ }^{18}$ überwiegt in Publikationen verständlicherweise vor allem aus der Hand von Verantwortlichen und Mitarbeitenden digitaler Editionen das uneingeschränkte Lob digitalen Edierens (siehe dazu unten). ${ }^{19}$ Allerdings findet die Entwicklung der digitalen Editorik unter einem nicht unerheblichen Druck der Förderinstitutionen statt, so dass es geboten ist, die Argumentationen kritisch zu prüfen, damit anerkannte editionsphilologische Konzepte der Text(re)präsentation und Kommentierung mit den neuen technischen Anforderungen harmoniert werden können.

\section{Kommentierung in gedruckten Briefeditionen}

Die Kommentierung von Bitzius' Korrespondenzen wird sich an den bisher im Rahmen der $H K G$ erarbeiteten Kommentarbänden, die separat zu den mit einem textkritischen Apparat ausgestatteten Textbänden erschienen sind, orientieren. ${ }^{20}$ In den gedruckten Kommentarbänden sind Angaben zur Materialität (Zeugenbeschreibung und Provenienz) und der Editorische Bericht untergebracht, der die Richtlinien für Text

15 Claudia Bamberg: „Schreiben Sie mir ja über alles“. Wozu eine digitale Edition der Korrespondenz August Wilhelm Schlegels? In: Vom Nutzen der Editionen. Zur Bedeutung moderner Editorik für die Erforschung von Literatur- und Kulturgeschichte. Hrsg. von Thomas Bein. Berlin u. a. 2015 (Beihefte zu editio. 39), S. 19-26, hier S. 20.

16 Siehe z. B. Digitale Briefedition Alfred Escher. Hrsg. von Joseph Jung. Zürich (https://www.briefedition. alfred-escher.ch/briefe/; Version: Juli 2015). Vgl. Vera Hildenbrandt und Roland S. Kamzelak: „Im Exil erweitert sich die Welt“. Neue Zugangswege zu Korrespondenzen durch Visualisierung. In: editio 28, 2014, S. 174-191.

17 Siehe Sahle 2013 (Anm. 8), 2, S. 162-164, und Sahle 2017 (Anm. 7), S. 240, 243 und 247.

18 Siehe z. B. Leuschner 2013 (Anm. 11), S. 226f., Wilhelm G. Jacobs: Überlegungen zum Medienwandel. In: editio 24, 2010, S. 14-22, hier S. 21, und Joachim Veit: Die Codierung digitaler Briefeditionen mit TEI P5. Konzepte und Probleme am Beispiel der Carl-Maria-von-Weber-Briefausgabe. In: Digitale Edition zwischen Experiment und Standardisierung. Musik - Text - Codierung. Hrsg. von Peter Stadler und Joachim Veit. Tübingen 2009 (Beihefte zu editio. 31), S. 217-232, hier S. 230f. Siehe auch den Beitrag von Ursula Kocher im vorliegenden Band.

19 Siehe z. B. Bamberg 2015 (Anm. 15) und Jochen Strobel: Digitale Briefedition und semantische ErschlieBung. Von den Briefen der Jenaer Romantikergeneration zur Edition der Korrespondenz August Wilhelm Schlegels. In: editio 28, 2014, S. 151-174.

20 Historisch-kritische Gesamtausgabe der Werke und Briefe von Jeremias Gotthelf. Hrsg. von Barbara Mahlmann-Bauer und Christian von Zimmermann. Hildesheim u. a. $2012 \mathrm{ff}$. 
und Kommentar sowie Angaben zur Überlieferungsgeschichte enthält. Zeitgenössische Quellen zu Entstehung und Rezeption der edierten Texte werden in Materialanhängen wiedergegeben, tabellarische Übersichten etwa zu gängigen Münzen oder chronologische Tafeln dienen der Entlastung des Kommentars. Register und je nach Abteilung Regesten erleichtern Forschenden die Arbeit mit dem edierten Material. Kern der Kommentarbände sind einerseits umfangreiche Überblickskommentare, welche den edierten Textbestand in den zeitgenössischen Kontexten verorten sowie die Entstehungsund Rezeptionsgeschichte aufarbeiten, andererseits umfassende Stellenerläuterungen zum Text. Letztere enthalten sprachliche und realienkundliche Erklärungen und legen intertextuelle Bezüge sowie Anspielungen auf Ereignisse und Diskussionen offen, die etwa in zeitgenössischen Zeitungen und anderen Druckerzeugnissen ihren Niederschlag fanden. ${ }^{21}$

Eine so umfassende Kommentierung, die auch die projektierte Korrespondenzedition beabsichtigt, ist freilich nicht unwidersprochen, hat sich aber bei gedruckten Briefausgaben in den letzten zwanzig Jahren als Standard durchgesetzt. Schon Winfried Woesler hat in seinen ,Vorschlägen für die Kommentargestaltung von Briefwechseln“22 auf der Grundlage seiner Arbeit an der Droste-Ausgabe den Standpunkt vertreten, die Erläuterungen müssten ,, alle Informationen, die zu einem umfassenden Verständnis der Brieftexte notwendig“" seien, enthalten, hat gleichzeitig aber den Kommentierenden anderer Briefeditionen den nötigen Spielraum zugestanden: ,[d]er Umfang ist Ermessenssache“. ${ }^{23}$ Auch wenn es im Einzelnen Unterschiede in der Kommentierungstiefe und -breite gibt, ist den wissenschaftlichen Briefausgaben eine Kommentierung gemeinsam, die über die bloße Identifizierung von Personen, Orten und Werken hinausgeht. So erklären die Stellenerläuterungen in der von Hans Zeller begründeten Edition C. F. Meyers Briefwechsel neben anderem ,historische Fakten spezieller Art, die nicht als selbstverständlich bekannt vorausgesetzt werden können; Personen und biographische Zusammenhänge; Sprachgebrauch, der infolge Sprachwandels zu Mißverständnissen führen kann“, um so das „Verstehen des Textes“ zu befördern. ${ }^{24}$ Auch die neue Goethe-Briefedition reicht Wort- und Sacherklärungen dar, verweist auf zeitgeschichtliche Bezüge und berücksichtigt in übergreifenden Erläuterungen das „,sich verändernde[ ] persönlich-biographische[ ] Umfeld des Absenders ebenso wie d[ie] Persönlichkeit der Adressaten und deren Beziehungen zu Goethe“. ${ }^{25}$ Gabriele Radecke hat in der Edition des Briefwechsels zwischen Theodor Fontane und Bernhard von Lepel eine

21 Vgl. Barbara Mahlmann-Bauer: Funktionen des Kommentars. Erfahrungsbericht anlässlich der Edition von Jeremias Gotthelfs politischer Publizistik. In: Internationalität und Interdisziplinarität der Editionswissenschaft. Hrsg. von Michael Stolz und Yen-Chun Chen. Berlin 2014 (Beihefte zu editio. 38), S. 199-218, und Christian von Zimmermann: Vom Kommentieren. In: Ebd., S. 219-237.

22 Winfried Woesler: Richtlinienvorschläge für Briefkommentare. In: Wissenschaftliche Briefeditionen und ihre Probleme. Editionswissenschaftliches Symposion. Hrsg. von Hans-Gert Roloff. Berlin 1998 (Berliner Beiträge zur Editionswissenschaft. 2), S. 87-96, hier S. 87.

23 Woesler 1998 (Anm. 22), S. 88.

24 Conrad Ferdinand Meyer - Friedrich von Wyß und Georg von Wyß. Briefe 1855 bis 1897 . Hrsg. von Hans Zeller und Wolfgang Lukas. Bern 2004 (C. F. Meyers Briefwechsel. Historisch-kritische Ausgabe. 3), S. 229-257, hier S. 252.

25 Johann Wolfgang Goethe. Briefe. 23. Mai 1764 - 30. Dezember 1772. Text. Hrsg. von Elke Richter und Georg Kurscheidt (Johann Wolfgang Goethe. Briefe. Historisch-kritische Ausgabe. 1 I). Berlin 2008, S. XVIII. 
„systematische Auswertung der zeitgenössischen Quellen“ vorderhand von Zeitungen vorgenommen; ${ }^{26}$ die Erläuterungen in der Edition des Briefwechsels zwischen Fontane und Theodor Storm informieren ,über den Entstehungsprozess der poetischen Werke“ der Autoren und setzen Rezensionen in Beziehung zu den Briefen. ${ }^{27}$ Insgesamt bestätigen eine Vielzahl weiterer Briefeditionen den an den Besonderheiten von Korrespondenztexten und den Informationsbedürfnissen der Nutzer und Nutzerinnen orientierten Standard einer breiten und tiefen Kommentierung, etwa auch die Marburger Büchner-Ausgabe oder die Edition von Briefen Wilhelm von Humboldts. ${ }^{28}$

Gattungsbedingte Besonderheiten der Briefkommentierung sind auf den Charakter der Briefe als Zeugnisse einer mündlich wie schriftlich geführten Kommunikation zurückzuführen. So sind die Briefe vom spezifischen Wissenshorizont geprägt, den sich die Korrespondierenden teilen. Dieser schlägt sich in Anspielungen und Auslassungen nieder, zu denen frühere Briefe, Briefe Dritter oder Beilagen Aufschluss geben können. ${ }^{29}$ Die Auflösung solcher Leerstellen ist Aufgabe der Erläuterungen ${ }^{30}$ - zumindest im oben skizzierten Kommentierungsverständnis. Auch Briefpartnerschaften rücken ins Zentrum der Kommentierung, wenn sie in Überblickskommentaren charakterisiert oder Lexika der Korrespondierenden wie in der Droste-Ausgabe zur Verfügung gestellt werden. ${ }^{31}$ Für das Verständnis von Korrespondenzen erlangen zudem Aspekte der Materialität besondere Bedeutung, die teils durch die Textwiedergabe (zum Beispiel die räumliche Anordnung von Textbestandteilen) dargestellt sind, teils ihren Niederschlag in Zeugenbeschreibungen finden. So sind Frankatur und Stempel - oder deren Fehlen - aufschlussreich für die Rekonstruktion der Übermittlungsart oder für Datierungen; der Gebrauch eines wertvollen Briefpapiers kann Ausdruck der Wertschätzung für den Adressaten oder die Adressatin oder je nachdem situativ bedingt sein. ${ }^{32}$

Den in Kommentarbänden untergebrachten Editionsbestandteilen kommen unterschiedliche Aufgaben in Bezug auf die Edition und das Lesepublikum zu. Der Editorische

26 Theodor Fontane und Bernhard von Lepel. Der Briefwechsel. Kritische Ausgabe. Hrsg. von Gabriele Radecke. 2 Bde. Berlin und New York 2006, Bd. 2 (Schriften der Theodor Fontane Gesellschaft. 5.2), S. 889.

27 Theodor Storm - Theodor Fontane. Briefwechsel. Kritische Ausgabe. Hrsg. von Gabriele Radecke. Berlin 2011 (Storm-Briefwechsel. 19), S. LX-LXIII, hier S. LXIII.

28 Siehe z. B. Georg Büchner: Briefwechsel. Marburger Ausgabe. Hrsg. von Burghard Dedner, Tilman Fischer und Gerald Funk. 2 Bde. Darmstadt 2012, Bd. 2 (Georg Büchner: Sämtliche Werke und Schriften. Historisch-kritische Ausgabe mit Quellendokumentation und Kommentar. 10.2), und Wilhelm von Humboldt: Briefe. Historisch-kritische Ausgabe. Hrsg. und kommentiert von Philip Mattson. Berlin 2014ff.

29 Siehe Brigitte Leuschner: Kommentierende und kommentierte Briefe. Zur Kommentargestaltung bei Briefausgaben. In: Kommentierungsverfahren und Kommentarformen. Hamburger Kolloquium der Arbeitsgemeinschaft für germanistische Edition 4. bis 7. März 1992, autor- und problembezogene Referate. Hrsg. von Gunter Martens. Tübingen 1993 (Beihefte zu editio. 5), S. 182-187, und Gabriele Radecke: „Schließlich die Bitte, mir das M.S. baldmöglich wiederzusenden, da es brennt“. Zur Kommentarfunktion von Briefbeilagen am Beispiel von Theodor Fontanes Briefwechseln mit Bernhard von Lepel und Theodor Storm. In: Probleme des Kommentierens. Beiträge eines Innsbrucker Workshops. Hrsg. von Wolfgang Wiesmüller. Innsbruck 2014 (Innsbrucker Beiträge zur Kulturwissenschaft. Germanistische Reihe. 80), S. 131-146.

30 Woesler 1998 (Anm. 22), S. 93.

31 Vgl. Annette von Droste-Hülshoff: Historisch-kritische Ausgabe. Werke - Briefwechsel. Hrsg. von Winfried Woesler. Bde. VIII-XII. Tübingen 1987-2000.

32 Siehe Wolfgang Rasch, Wolfgang Lukas und Jörg Ritter: Gutzkows Korrespondenz-Probleme und Profile eines Editionsprojekts. In: Brief-Edition (Anm. 11), S. 87-107, hier S. 97-100, und Elke Richter: Goethes Briefhandschriften digital - Chancen und Probleme elektronischer Faksimilierung. In: Brief-Edition (Anm. 11), S. 53-74, hier S. 61. 
Bericht leistet die Legitimation sowie Offenlegung der gewählten Prinzipien und der Auswahl des edierten Materials. Für die Nutzer werden die editorischen Entscheide nachprüfbar und die Edition verständlich. Diskursive Texte wie Einleitungskommentare oder Essays zum edierten Material und Einzelstellenerläuterungen bieten Hinweise auf Kontexte und dienen der Verringerung der historischen Distanz, also der Vermittlung des edierten Texts. Insofern gehört auch der Abdruck zeitgenössischer Quellen der Entstehung und Rezeption sowie von Prätexten hierher. Der Kommentierung kommt folglich eine erschließende Funktion zu, die Grundlage jeder wissenschaftlichen Beschäftigung mit dem edierten Korpus und dessen Interpretation ist. ${ }^{33}$ In diskursiven Kommentaren oder Essays ist überdies bereits ,,der Übergang vom Kommentar zur eigenständigen Untersuchung angelegt" ${ }^{34}$

\section{Kommentierung in digitalen Briefeditionen}

Auf die genannten Bestandteile traditioneller Kommentarbände hat digitales Edieren vielfältige Auswirkungen. So machen die Qualitätsanforderungen an eine wissenschaftliche Edition eine Erweiterung des Metakommentars durch die Codierungsrichtlinien notwendig, und materiale Aspekte werden durch die Bereitstellung von Digitalisaten veranschaulicht, ${ }^{35}$ was jedoch - solange es keine bildinterpretierenden Programme gibt - kein Ersatz für eine explizite Codierung der Besonderheiten des Textzeugen sein kann.

Im Vergleich mit gedruckten Ausgaben sind die einzelnen Editionsbestandteile auf der digitalen Benutzeroberfläche alinear angeordnet. Metadaten, zu denen die klassische Zeugenbeschreibung zählt, sind wie etwa in der Schlegel-Edition nach Bedarf ein- oder ausblendbar, was auch die Präsentation entlastet. Teilweise können Nutzer und Nutzerinnen - so etwa auf der Benutzeroberfläche der Edition Briefe und Texte aus dem intellektuellen Berlin um $1800^{36}$ - zwischen Einzel- und synoptischer Ansicht wählen und sich letztere nach eigenen Interessen zusammenstellen. ${ }^{37}$ Die Stellenerläuterungen werden wie bei der Escher-Briefedition als Endnoten angeboten. In der digitalen Präsentation der van Gogh-Briefedition, die ebenfalls eine frei zusammenstellbare, drei- (bzw. mehr-)spaltige synoptische Ansicht vorsieht, können die Stellenerläuterungen konstant parallel zum edierten Text angezeigt oder in der Mittelspalte aufgerufen werden, wenn die entsprechende Erläuterungsnummer im Text angeklickt wird. Im Mark Twain Project hingegen ist eine feste zweigliedrige synoptische Ansicht vorgegeben, mit ediertem Text links und Stellenerläuterungen rechts, die wiederum in editorische und in erklärende Einträge unterteilt sind. Der Navigationserleichterung dient die farbliche Hervorhebung derjenigen Erläuterung, deren Nummer im edierten Text angeklickt wird. Die Carl-Maria-von-Weber-Gesamtausgabe (WeGA) schließlich

\footnotetext{
Siehe Leuschner 1993 (Anm. 29), S. 187.

Leuschner 1993 (Anm. 29), S. 186.

35 Siehe Anton Unterkirchner: Zur Materialität und Form von Korrespondenzen. In: Probleme des Kommentierens (Anm. 29), S. 97-113, hier S. 103, und Richter 2013 (Anm. 32), S. 65, S. 69 und S. 74.

36 Briefe und Texte aus dem intellektuellen Berlin um 1800. Hrsg. von Anne Baillot. Berlin (https://www. berliner-intellektuelle.eu/).

37 Siehe zur Einrichtung der ,editorische[n] Oberfläche“ durch die Nutzer auch Helbig 2016 (Anm. 7), S. 110.
} 
zeigt Stellenerläuterungen im Text als Popup sowie zusätzlich in der separaten Ansicht ,Apparat ${ }^{6}{ }^{38}$ Gewiss muss der synoptischen Anzeige von Text und Stellenerläuterungen der Vorzug gegenüber nachgestellten Anmerkungen gegeben werden, insbesondere bei längeren Briefen. Auch das Aufrufen von Stellenerläuterungen in einem Popup-Fenster ist nicht immer optimal, etwa wenn sich das Fenster - wie in vielen digitalen Ausgaben - direkt über dem Text öffnet und somit den Text um die erläuterte Textstelle verdeckt. Die Grenzen der synoptischen Darstellung liegen demgegenüber eher in der beschränkten Größe von Bildschirmen.

Doch nicht nur die Präsentation von Kommentarbestandteilen verändert sich in der digitalen Editorik. Auch die Inhalte von Stellenerläuterungen und Überblickskommentaren, die in gedruckten Briefausgaben grundlegende Bedeutung haben, wandeln sich.

Zunächst ist auffällig, dass sich etliche Fachbeiträge mit den Möglichkeiten digitaler Briefeditionen befassen, doch eher vage bleiben, was die Kommentierung anbelangt, oder sich deutlich vom Standard gedruckter Briefausgaben distanzieren. So beginnt Claudia Bamberg ihren Beitrag über die Digitale Edition der Korrespondenz August Wilhelm Schlegels ${ }^{39}$ zwar mit der Erkenntnis, ,zuverlässige und gut kommentierte Editionen" seien Voraussetzung für weiterführende Fragestellungen, ${ }^{40}$ kommt jedoch bald zum Schluss: „Mit der Möglichkeit der digitalen Edition von Briefen ändert sich nun aber fast zwangsläufig die Vorstellung dessen, was eine ,zuverlässige und gut kommentierte 'Edition sei.“41 Keineswegs sollten „die philologischen Standards über Bord geworfen werden“, und am Ende des Projekts müsse ,immer ein sorgfältig redigiertes Ergebnis stehen“, auch wenn Editionsbestandteile bereits zu einem früheren Zeitpunkt - freilich versehen mit einer Angabe zum Status (etwa ,transkribiert") - bereitgestellt würden. ${ }^{42}$ Doch was ist gemäß dieser Vorstellung eine ,gut kommentierte“ Edition im digitalen Zeitalter?

Gemeint ist vielmehr, dass sich im digitalen Medium die Möglichkeiten der Erschließung und Kommentierung von Briefen geändert haben und dass dies auch Auswirkungen auf die Präsentation und Nutzung einer Web-Briefedition hat. An die Stelle des konventionellen Stellenkommentars treten neue Formen der Auszeichnung und der Registererstellung, neue Konzepte der semantischen Erschließung sowie zahlreiche Möglichkeiten der Visualisierung. ${ }^{43}$

Überlegungen zur semantischen Erschließung, ,deren Konturen sich noch nicht klar abzeichnen" würden, hat für die Schlegel-Edition Jochen Strobel vorgestellt. ${ }^{44}$ Demnach solle diese Erschließung mit einer konkreten Forschungsfrage verknüpft sein und von einer Inhaltsanalyse ausgehen, um zu einem aussagekräftigen Thesaurus zu gelangen. ${ }^{45}$ Die semantische Erschließung müsse als ,zusätzlich strukturierende Größe“

\footnotetext{
38 Carl-Maria-von-Weber-Gesamtausgabe. Digitale Edition (http://www.weber-gesamtausgabe.de/; Version 3.2.1: 8. Januar 2018).

39 Digitale Edition der Korrespondenz August Wilhelm Schlegels (http://august-wilhelm-schlegel.de/).

40 Bamberg 2015 (Anm. 15), S. 19.

41 Bamberg 2015 (Anm. 15), S. 20.

42 Ebd.

43 Ebd.

44 Strobel 2014 (Anm. 19), S. 151.

45 Siehe Strobel 2014 (Anm. 19), S. 170-172.
} 
Eingang in digitale Briefeditionen, die große Datenmengen bereitstellen, finden. ${ }^{46}$ Eine Kommentierung, wie sie sich als Standard in Druckeditionen durchgesetzt hat, ist in der Schlegel-Edition - zumindest legen dies die Publikationen nahe - nicht vorgesehen. Vielmehr hält Strobel fest, mit dem gewählten Verfahren entferne man sich ,vom Credo einer auf historisch-kritische Vollständigkeit beharrenden Editionswissenschaft“ ${ }^{47}$ Erschließung also statt (historisch-kritische) Kommentierung?

Eine Sichtung digitaler Briefeditionen mag die gegenwärtige Praxis erhellen. Hierfür wurden mehrheitlich Editionen aus dem deutschsprachigen Raum ausgewählt, die unterschiedliche Ansprüche und Ausrichtungen haben und aktuell auch nicht alle fertiggestellt sind. Größtenteils sind sie TEI- oder zumindest XML-konform.

Gerade die abgeschlossenen digitalen Briefeditionen größeren Umfangs, die auch (oder ursprünglich ausschließlich) als gedruckte Editionen konzipiert wurden, enthalten zum Teil umfangreiche Stellenerläuterungen und einführende Texte zum edierten Briefkorpus. Hier ist etwa an die Briefedition im Rahmen des Mark Twain Projects, ${ }^{48}$ an die Edition Vincent van Gogh - The Letters ${ }^{49}$ oder an die Alfred Escher-Briefedition $^{50} \mathrm{zu}$ denken. Letztere stellt zum Beispiel für eine Auswahl von Briefen, die auch in der gedruckten Ausgabe ediert wurden, Stellenerläuterungen zur Verfügung. Die Überblickskommentare bilden nicht nur eine inhaltliche Klammer um die Briefe, sondern haben den Charakter weiterführender Forschungsarbeiten, welche das Briefkorpus auswerten wie etwa hinsichtlich der Thematisierung des Gotthardbahnprojekts. ${ }^{51}$ Für die Kommentierung orientiert sich die Escher-Briefedition am Standard gedruckter Briefausgaben:

Die weiterführend kommentierten Briefe verfügen [...] über Kommentare, welche Informationen enthalten, die zur Kontextualisierung beitragen. Darin finden sich etwa biographische Angaben zu erwähnten Personen, Erläuterungen zu Sachverhalten, erwähnten Dokumenten, technischen Begriffen sowie zu lateinischen oder nicht mehr gebräuchlichen deutschen Begriffen, Quellenausweise sowie Verweise auf andere Briefe und auf Überblickskommentare. ${ }^{52}$

Andere Editionen bieten Stellenerläuterungen, die sich mehrheitlich auf den Verweis anderer in der Edition aufgearbeiteter Dokumente (etwa Briefe, Tagebücher, Werke) zurückziehen, und Überblickskommentare unterschiedlicher Länge. So verfährt etwa das Darwin Correspondence Project, das Stellenerläuterungen zu einem Teil der rund 9000 zur Verfügung gestellten Schreiben und unter der Rubrik , commentary ' diskursive Kommentare unterschiedlicher Länge zu einzelnen Themen bietet. ${ }^{53}$ Nicht alle diskursiven Kommentare sind im Projekt entstanden, so wird etwa ein älterer Artikel

46 Strobel 2014 (Anm. 19), S. 155.

47 Strobel 2014 (Anm. 19), S. 153.

48 Mark Twain Project (http://www.marktwainproject.org).

49 Vincent van Gogh. The Letters (http://vangoghletters.org/vg/).

50 Digitale Briefedition Alfred Escher (Anm. 16).

51 Vgl. etwa die Aufsätze von Joseph Jung, Lisa Bollinger und Vincent Pick in: Alfred Eschers Briefwechsel 1866-1882. Private Eisenbahngesellschaften in der Krise, Gotthardbahn, politische Opposition. Hrsg. von Joseph Jung im Auftrag der Alfred Escher-Stiftung. Bd. 6. Zürich 2015; Digitale Briefedition Alfred Escher (Anm. 16; https://www.briefedition.alfred-escher.ch/kontexte/uberblickskommentare/).

52 Digitale Briefedition Alfred Escher (Anm. 16; https://www.briefedition.alfred-escher.ch/uber-die-edition/ editionsprinzipien/).

53 Darwin Correspondence Project (https://www.darwinproject.ac.uk). 
zu Darwins Position in der Debatte um Tierversuche re-publiziert und mit Links auf die im Beitrag erwähnten und in der Edition zur Verfügung gestellten Dokumente versehen. ${ }^{54}$ Auch die Carl Weber-Gesamtausgabe (WeGA) bietet seit dem Release 3.0 Themenkommentare unterschiedlichen Umfangs mit äußerst gewinnbringenden, auf Briefen und Tagebuchaufzeichnungen basierenden Erläuterungen, etwa zur Mitwirkung Webers am Prager Ständetheater. ${ }^{55}$ Während manche Stellenerläuterungen durchaus weiterführenden Charakter haben und etwa auf Besprechungen aufgeführter Stücke verweisen, ${ }^{56}$ beschränkt sich die Mehrheit auf Hinweise auf relevante Tagebuchstellen oder Passagen aus andern edierten Briefen. Freilich hätte sich die Leserin an manchen Stellen eine zusätzliche Erläuterung gewünscht, etwa zum Brief von Weber an Caroline Brandt vom 7. Januar 1817. Darin bemerkt Weber, er könne Anton Schmidl manches verzeihen, und dieser habe ihm angeboten, bei ihm zu wohnen. ${ }^{57}$ Unklar bleibt, was Schmidl verziehen werden müsste, ebenso was es mit dem Wohnungsangebot auf sich habe. Allerdings bedeutet der Status, bearbeitet' in der $W e G A$ eben auch nur, dass die Texte ,,mit den augenblicklich möglichen Anmerkungen versehen" sind und sich Kommentare wohl noch verändern können. ${ }^{58}$ Diese prinzipielle Offenheit für Ergänzungen, welche der unabgeschlossene Status digitaler Editionen ermöglicht, führt gelegentlich zu Irritationen. Vielleicht wäre anzuraten, die Lemmatisierung jener Textstellen bereits auszuweisen, deren Erläuterung noch erarbeitet wird. Dies erleichterte nicht nur die kritische Begutachtung, sondern ermöglichte auch die Mitwirkung von Nutzenden, die vielleicht Hinweise geben können.

Viel wichtiger scheint für die vorliegende Frage jedoch die Beobachtung, dass in einigen, gerade größeren digitalen Briefeditionen Stellenerläuterungen ganz fehlen und dafür eine wie auch immer geartete (semantische) Erschließung geboten wird. So ermöglichen die einführenden Kommentare auf Burckhardt Source - einer insgesamt ansprechend gestalteten Edition von Briefen an Jacob Burckhardt - thematische Zugänge zum Briefkorpus, die gewiss nicht den gleichen Anspruch wie die diskursiven Texte der Escher-Briefedition haben. Die unterschiedlich langen Kommentare bilden inhaltliche Klammern um ein Teilkorpus und heben die Bedeutung einzelner Dokumente für den Themenbereich hervor. In „Europe. Historical facts in everyday life" erhalten etwa die Ereignisse im Zuge des Risorgimento in Italien einigen Raum.

54 Siehe https://www.darwinproject.ac.uk/commentary/life-sciences/darwin-and-vivisection/ - Weitere Beispiele mit Kommentaranteil, die nicht den Anspruch haben, alle Briefe gleich ausführlich zu erläutern oder diskursive Kommentare bereitzustellen, sind etwa: Briefe und Texte aus dem intellektuellen Berlin um 1800 (Anm. 36) und: Correspondance entre Henri Fantin-Latour et Otto Scholderer (1858-1902). Édité par Mathilde Arnoux, Thomas W. Gaehtgens et Anne Tempelaere-Panzani. Édition critique en ligne 2014 (http://quellen-perspectivia.net/fr/fantin-scholderer/start).

55 Siehe z. B. Franz Ziegler: Der Aufbau des deutschsprachigen Musiktheater-Ensembles am Prager Ständetheater, und ders.: Das Prager Ständetheater zu Beginn des 19. Jahrhunderts. Beide in: Carl-Maria-vonWeber Gesamtausgabe (Anm. 38; http://www.weber-gesamtausgabe.de/de/A009005/Themenkommentare/ A090005.html; http://www.weber-gesamtausgabe.de/de/A009005/Themenkommentare/A090071.html).

56 Vgl. Brief von Weber an Johann Baptist Gänsbacher vom 27. Februar 1811, Lemma ,in Berlin aufgeführt werden soll“ (http://weber-gesamtausgabe.de/A040382; Version 3.2.1 vom 8. Januar 2018).

57 http://weber-gesamtausgabe.de/A041003 (Version 3.2.1 vom 8. Januar 2018).

58 Editionsrichtlinien zur Ausgabe der Briefe, Tagebücher und Dokumente Webers. In: Carl-Maria-von-WeberGesamtausgabe (Anm. 38; http://www.weber-gesamtausgabe.de/de/Projekt/Editionsrichtlinien_Text.html). 
[...] Two more letters by Commeter [Johann Matthias, Kunsthändler] and another one by Jenison [Franz Oliver Graf von, Diplomat], sent in 1861, inform the readers about Italian current events after Garibaldi's expedition. In particular, Jenison's letter, sent on January 22nd 1861, is noteworthy: here Jenison expresses some doubts [...] about the general enthusiasm of the Italian youth. Jenison concludes asking Burckhardt whether the nationalism could help European development and culture. ${ }^{59}$

Dagegen verweist „Music. A florilegium of melodies“ in fünf Sätzen bloß auf die Musikleidenschaft Burckhardts und charakterisiert das zugehörige Korpus als Briefe von Freunden mit der gleichen Leidenschaft: „For this reason the collection is characterised by familiar notes and casts light on more private aspects." 60

Auf Stellenerläuterungen verzichtet Burckhardt Source wie wohl auch die Schlegel-Edition ganz.

\section{Stellenerläuterungen und Entitäten}

Claudia Bambergs Äußerung zum Konzept der Schlegel-Edition verweist darauf, dass vielfältige Erschließungen und Visualisierungen traditionelle Stellenerläuterungen ablösen. In eine ähnliche Richtung zielen die Editionsrichtlinien der $W e G A$ :

Viele Aufgaben des früheren Einzelstellenkommentars (z. B. die Auflösung verdeckter Namen, von Kose- oder Scherznamen, von Pseudonymen, Verweisen auf Werke oder Rollenangaben, Hinweise auf Zeitungsartikel oder andere Schriftstücke) können nun allerdings durch eine direkte inhaltliche Auszeichnung des Textes entfallen $[\ldots] .{ }^{61}$

Die Codierung von Personen, Orten und Werken als Entitäten (etwa mittels der TEI-Elemente $<r s>$ oder <persName>) sowie deren Verknüpfung mit meist erläuternden Registern übernimmt also die der klassischen Stellenerläuterung vorbehaltene Identifikation von Personen, Orten und Werken, auf die in den edierten Briefen nur angespielt wird. Die genannte Codierung erlaubt nicht nur die Erzeugung eines Registers für die digitale Präsentation oder den Druck, sondern auch die Anzeige von Personennamen durch Anklicken im Brieftext und die Bereitstellung der dazugehörigen, erläuternden Registereinträge. In der $W e G A$ sind die betreffenden Passagen hervorgehoben; beim Anklicken öffnet sich ein Fenster mit je unterschiedlich langen Hinweisen zur Entität. Die Edition von Vincent van Goghs Briefen und die Escher-Briefedition identifizieren dagegen eine nur andeutungsweise erwähnte Person in den Stellenerläuterungen (in Form eines Freitexts) und codieren sie zusätzlich als maschinell auswertbare Entität. ${ }^{62}$ Denkbar ist, dass diese Doppelcodierung funktional begründet ist und sich die Herausgeber so eine flexible Gestaltung der Stellenerläuterungen (für den Druck) sichern wollten, die es erlaubt, eine von der allgemeineren Entitätencodierung unabhängige, auf die konkrete Einzelstelle zugeschnittene Erläuterung anzubieten.

In der $W e G A$ werden zudem ganz unterschiedliche, auch lexikalische Informationen zu Personen, Orten und Werken bereitgestellt. Bei Personen sind es beispielsweise

59 Burckhardt Source (http://burckhardtsource.org/collection/5).

$60 \mathrm{http}: / /$ burckhardtsource.org/collection/3/.

61 Editionsrichtlinien zur Ausgabe der Briefe, Tagebücher und Dokumente Webers (Anm. 58).

62 Siehe z. B. Erläuterung zu Eduard in: To van Gogh, Theo, 17th March 1873. In: Vincent van Gogh (Anm. 49; http://vangoghletters.org/vg/letters/let005/letter.html). 
immer die sogenannten ,Basisdaten', eine tabellarische Liste von Alternativnamen, Lebensdaten, Berufen und Wirk- oder Wohnorten, die beim Anklicken auf die betreffende Entität angezeigt werden. Im Registersatz zu einer Person finden sich diese wieder, allenfalls werden Bilder unter ,Ikonographie“ angezeigt und unter ,Biographien“ sind weiterführende Dokumente anwählbar. Zu diesen zählen der ,WeGA Text ${ }^{\star}$, biographische Angaben, die im Projekt zusammengestellt werden und über die Basisdaten hinausgehen, aber auch Wikipedia- und ADB-Artikel, die trotz ihrer externen Herkunft innerhalb der projekteigenen Benutzeroberfläche geladen werden. ${ }^{63}$ Auf externe Websites werden die Nutzerin und der Nutzer über die ,PND Beacon Links' geleitet. Basisdaten, ,WeGA Text` und GND sind in der XML-Datei zu einer Person abgelegt; die Webanwendung steuert, welche externen Ressourcen dynamisch nach der fraglichen GND abgesucht und in der digitalen Präsentation angezeigt werden sollen. ${ }^{64}$

Bei Burckhardt Source kann die Codierung von Entitäten zu Personen, Orten und (Kunst-)Werken, die im Text implizit oder explizit erwähnt sind, durch die Nutzerin bei Bedarf in der sogenannten semantischen Ansicht sichtbar gemacht werden. Der Text der externen Ressource zu einer Entität wird samt Quellennachweis angezeigt. Die Bearbeiter und Bearbeiterinnen haben hierfür Links auf möglichst aussagekräftige Ressourcen aus digitalen Bibliotheken annotiert; fehlten solche, trugen sie die Informationen selbst zusammen und stellten sie auf externen Plattformen andern wieder zur Verfügung. ${ }^{65}$ Freilich können die Links auf externe Ressourcen nicht direkt aus Burckhardt Source aufgerufen werden. Ebenso führen die unter „Original source“ angegebenen Links zu leeren Seiten. Möglicherweise wird diese Unzulänglichkeit behoben, denn bereits in der Vergangenheit bot die Edition aufgrund technischer Umzüge vorübergehend nicht alle Ansichten an. Dieses grundsätzliche Problem digitaler Ressourcen - die Abhängigkeit der Präsentation von technischen oder infrastrukturellen Bedingungen - beeinträchtigt die Zitierfähigkeit und wirkt auch qualitätsvermindernd. Überhaupt wünscht man sich bei vielen Editionen leicht zugängliche Informationen zum Arbeitsstand und zum Stand der realisierten und geplanten (vielleicht auch aufgegebenen) Funktionalitäten.

Die zwei Beispiele verdeutlichen die Erweiterung der Vernetzungsmöglichkeiten, mitunter wie bei der WeGA durch den Einsatz standardisierter Normdateien. Die Vorzüge der Vernetzung sind in der Forschungsgemeinschaft gewiss zu Recht unumstritten. Auch die digitale Gotthelf-Gesamtedition wird zu Personen mit Hilfe von GND und Skripts im Register Links auf externe Ressourcen anbieten. Dabei sind nicht nur digital zugängliche Lexika wie das Historische Lexikon der Schweiz oder die Deutsche Biographie relevant, sondern auch digitale Editionen, in denen Informationen zu einer Person zu finden sind. Beispielsweise kann ein Link auf die Gesamtausgabe der Briefe Friedrich Fröbels verweisen, in der ein Brief Fröbels an Bitzius sowie mehrere Briefe

63 Siehe Joachim Veit: Es bleibt nichts, wie es war - Wechselwirkungen zwischen digitalen und ,analogen ' Editionen. In: editio 24, 2010, S. 37-52, hier S. 46.

64 Vgl. Peter Stadler: Normdateien in der Edition. In: editio 26, 2012, S. 174-183, hier S. $177 \mathrm{f}$.

65 Costanza Giannaccini und Susanne Müller: Burckhardtsource.org: A Semantic Digital Library. In: AIAUCD, 14. Proceedings of the Third AIUCD Annual Conference on Humanities and Their Methods in the Digital Ecosystem. New York 2015 (http://dl.acm.org/citation.cfm?id=2802631). 
Fröbels ediert sind, in denen er gegenüber Dritten über Bitzius schreibt. ${ }^{66}$ Die Zuweisung von Normdateien ist darüber hinaus Voraussetzung für die Berücksichtigung von Editionsdaten in Archivkatalogen und Suchportalen, wodurch sich die Möglichkeiten der Erschließung der Edition immens erweitern, da „die einmal gewonnenen Erschließungsdaten $[\ldots]$ auch in neuen Umgebungen verwertbar“ sind. ${ }^{67}$

Dieser Punkt berührt die Frage der über eine bloße Lektüre hinausgehenden Nachnutzung von Editionsmaterial. Für die Verwaltung von für eigene Forschungsfragen aufschlussreichen Briefen (und Kommentaren) können in der Escher-Briefedition Benutzerkonti eingerichtet werden; eine direkte Nachnutzung der downloadbaren XML-Dateien steht Interessierten in der WeGA oder der Edition von Briefen und Werken im intellektuellen Berlin um 1800 offen. Letztere ermöglicht auch den Download von PDF-Dateien, in denen allfällige Stellenerläuterungen und Register (inklusive verzeichneter Entitäten) mitgeliefert werden. Das dem Wiener Musiktheoretiker gewidmete Projekt Schenker Documents Online stellt PDF- und ePub-Dateien zur Verfügung, in denen die ausgezeichneten Entitäten aufgeschlüsselt werden. ${ }^{68}$ Schließlich deutet die Verknüpfung von Benutzeroberflächen mit Annotationsumgebungen - wie etwa bei Burckhardt Source - auf die Entwicklung hin, die digitale Editionen als Teil von Forschungsplattformen definiert, auf denen Nutzer an der Edition - sowohl im Bereich der Textphilologie als auch der Kommentierung - mitarbeiten können und sollen. ${ }^{69}$

Stellenerläuterungen und Entitätencodierung machen dennoch zwei Nachfragen notwendig. Die erste betrifft die Anzeige externer Ressourcen direkt in der digitalen Präsentation wie etwa in der $W e G A$. Diese Informationen, die ja nicht in der Edition erarbeitet worden sind, fehlen folgerichtig in den XML-Dateien. Nur die Webanwendung bewirkt deren Anzeige. Gleichwohl fragt sich die im Buchzeitalter sozialisierte Leserin, welchen Status diese Informationen für die Gesamtedition haben, wenn sie als Volltext in die digitale Präsentation der Edition Eingang finden. Erhalten sie damit qualitativ nicht einen anderen Charakter als bloße Links, die dem Leser und der Leserin den Weg zum entsprechenden Zielpunkt weisen?

Von größerer Tragweite ist der zunehmende Verzicht auf Stellenerläuterungen. Natürlich übernimmt die Codierung von Personen, Orten und Werken als Entitäten dank der Verbindung mit einem editionsweiten zentralen Register die identifizierende Aufgabe von Stellenerläuterungen. Zudem haben weiterführende Registereinträge oder angezeigte Informationen erläuternden Charakter und werden zum Beispiel in der WeGA wie Stellenerläuterungen als Popup realisiert. Für eine erste und generelle Orientierung mögen solch lexikalische Informationen - mitunter aus externen Ressourcen - hilfreich sein, die editionsseitig ohne größeren Aufwand bereitgestellt werden können, sofern dies wie bei der $W e G A$ über ein maschinelles Abfragen über Normdateien geschieht. Freilich kann auch die Selektion hinterfragt und der Qualitätsanspruch der beigezogenen

\footnotetext{
66 Personen G. [Personenregister]. In: Gesamtausgabe der Briefe Friedrich Fröbels (https://editionen.bbf.dipf.de/ briefedition-friedrich-froebel/personenregister/personenregister-g\#JG4).

67 Jutta Weber: Briefnachlässe auf dem Wege zur elektronischen Publikation. Stationen neuer Beziehungen. In: Brief-Edition (Anm. 11), S. 25-33, hier S. 31.

68 Schenker Documents Online (http://www.schenkerdocumentsonline.org/index.html).

69 Siehe Strobel 2014 (Anm. 19), S. 152.
} 
Ressourcen wie beispielsweise Wikipedia geklärt werden. ${ }^{70}$ Wenn eine Verlinkung mit externen Ressourcen für eine generelle Erläuterung von Personen durchaus sinnvoll scheint, ist dies bei der Erläuterung von Orten, die ja im Unterschied zu Personen mit begrenzter Lebenszeit meist fortbestehen, nicht automatisch gegeben. Auf Burckhardt Source sind etwa etliche Briefe ediert, in denen von der Heimatstadt Jacob Burckhardts die Rede ist. In allen Briefen, die ja aus dem 19. Jahrhundert stammen, wird zur Entität Basel eine Charakterisierung der Stadt in der Gegenwart der 2010er Jahre geboten. ${ }^{71}$ Diese Information, die die Nutzerin auch sonst problemlos im Internet abrufen kann, erhellt die Bedeutung Basels zur Entstehungszeit der Briefe nicht. Hingegen wären hierfür eher Angaben zur Größe, Bevölkerungszahl, zur politischen und wirtschaftlichen Lage der Stadt im 19. Jahrhundert gewinnbringend, gerade weil sich Basel nicht nur seither, sondern auch in den beinahe fünfzig Jahren, welche die Korrespondenzen abdecken, stark verändert hat. Die Nichtberücksichtigung des historischen Abstandes führt zu belanglosen, wenn nicht gar falschen Informationen für eine Erläuterung der konkreten Textstelle.

Sowohl bei Personen als auch bei Orten können Informationen, die externen Ressourcen entstammen oder lexikalischen Charakter haben, nur allgemeine und unspezifische Informationen bieten. Eine konkrete, unklare oder zweideutige Stelle im Editionstext kann damit nicht erläutert werden.

\section{Zum Wert einer umfassenden Kommentierung}

Letztlich führt dies zur Frage, wie weit die Aufarbeitung von Kontexten gehen soll und was die Kommentierung im engeren Sinn leisten muss. Dies hat nur bedingt mit dem Erfassungs- und Präsentationsmedium zu tun, auch wenn gerade hinsichtlich der Darstellung längerer Texte und von Stellenerläuterungen noch Optimierungsbedarf besteht. Die Möglichkeiten des digitalen Mediums begünstigen aktuelle Forschungsfragen wie Netzwerkanalysen oder quantifizierende Untersuchungen. In dieser Perspektive kann die Aufgabe der Edition darin liegen, die hierzu nötigen Grundlagen bereitzustellen. Die Hoffnung einiger Editoren und Editorinnen besteht offenbar darin, dass der Verlust, den ein Verzicht auf eine (weitergehende) Kommentierung mit sich bringt, durch die vielfältigen Möglichkeiten der Erschließung und Vernetzung aufgewogen werden kann; zudem ist der Verzicht gewiss in finanzieller Hinsicht günstig. ${ }^{72}$

Doch so sorgfältig die Erschließung auch sein mag, sie ist in keiner Weise vergleichbar mit einer sorgfältigen Kommentierung, die mit der Aufarbeitung von Kontexten über den einzelnen Text hinausweist. Eine so umfassende inhaltliche Erschließung verlangt von Kommentatoren profunde Kenntnisse der historischen Umstände und der zeitgenössischen Debatten. Zudem wäre es schwierig, mit einer Verlinkung auf externe Ressourcen mehrere mögliche Quellen und Anspielungen einer konkreten Textstelle offenzulegen und diese nach Relevanz-, Plausibilitäts- oder weiteren Kriterien in ein Verhältnis zueinander und zu der Textstelle zu

70 Vgl. Angela Steinsiek: Alles Wikipedia? Kommentieren heute am Beispiel der Jean Paul-Brief-Edition. In: Brief-Edition (Anm. 11), S. 229-235.

71 Siehe z. B. Brief Nr. 702 von Jakob Kuhn, 6. Oktober 1857 (https://burckhardtsource.org/letter/702?semantic).

72 Siehe etwa Strobel 2014 (Anm. 19), S. 153. 
bringen. Dies vermag eigentlich nur eine Erläuterung adäquat zu leisten. ${ }^{73}$ Großprojekte wie die WeGA oder die Schlegel-Edition beabsichtigen keine breite Kommentierung, zumindest legen dies die publizierten Editionsrichtlinien nahe. Es sei „nach Konzepten gesucht“" worden, wie die ,Verflechtung der Texte und ihre Kommentierung dem Leser mit ökonomischem Einsatz der Ressourcen und dennoch möglichst effektiv zu vermitteln wären“. ${ }^{74}$ Die digitale Publikation solle ,den Aufwand für die Kommentierung in Grenzen" halten. ${ }^{75}$

Auch die Edition von Albert Bitzius' Korrespondenzen wird nicht alle edierten Briefe breit kommentieren, sondern orientiert sich am Modell der Escher-Briefedition und wird vorderhand nur jene Briefe umfassend kommentieren, die auch in der gedruckten Ausgabe erscheinen. Um den Zugang zu allen digital präsentierten Briefen zu erleichtern, werden sie jedoch mit Schlagwörtern erschlossen, Personen, Orte und Werke werden als Entitäten ausgezeichnet. Darauf aufbauend können zu einem späteren Zeitpunkt die übrigen Briefe ebenfalls umfangreich kommentiert werden. Diese inhaltlichen Auszeichnungen werden in der digitalen Präsentation in der Leseansicht zugänglich sein, während weitere Ansichten die Textgenese ins Zentrum rücken. Die Einrichtung unterschiedlicher Nutzungsszenarien beugt einer Überfrachtung der Präsentation vor und macht die Edition so erst sinnvoll benutzbar. Kombinierbare Such- und Filterfunktionen werden zur Unterstützung der Nutzung ebenso zur Verfügung gestellt wie Visualisierungen oder Zeitleisten. Insgesamt sollen die vielfältigen Vorzüge digitalen Edierens zum Tragen kommen, ohne dabei den Kommentierungsanspruch historisch-kritischer Editionen zu vernachlässigen. Schließlich kann gerade eine digitale Präsentation wesentlich dazu beitragen, die Leistungen von Editionen nicht nur im Bereich der Textphilologie, sondern auch der Kommentierung sichtbarer zu machen und so im öffentlichen Bewusstsein zu verankern.

Der Entscheid, keine oder nur eine begrenzte Anzahl von Erläuterungen anzubieten, scheint in Zeiten knapper finanzieller Ressourcen als pragmatischer Ausweg plausibel. Gleichwohl ist er wissenschaftspolitisch dann problematisch, wenn er mit dem der Legitimation dienenden Versuch verbunden ist, eine Erschließung als Ersatz für eine sorgfältige Kommentierung zu propagieren, obwohl es sich hierbei um zwei unterschiedliche Dienst- und Forschungsleistungen handelt. Der Nutzen einer breit kommentierenden Edition, die wie die HKG Jeremias Gotthelf Bezüge zu zeitgenössischen Kontexten aufdeckt, liegt jenseits der Lektüre durch interessierte Leserinnen und Leser gerade darin, dass Diskurse aufgearbeitet werden, in denen die edierten Texte angesie-

\footnotetext{
73 Janine Droese und Andreas Münzmay stellen für ihr Untersuchungsobjekt fest, dass der , historische Link in Texten oft ,nicht ein bestimmtes, genau definierbares Ziel ansteuert, sondern als ein eher mehrdimensionales Gebilde auf ein weites Feld an Wissensmöglichkeiten führt, die ihrerseits wieder in eigenen Kontexten stehen": Pfade im editorischen Netz. Überlegungen zur Pragmatik des editorischen Hyperlinks am Beispiel der Comédie en vaudevilles Annette et Lubin (1762). In: editio 29, 2015, S. 85-102, hier S. 100.

74 Editionsrichtlinien zur Ausgabe der Briefe, Tagebücher und Dokumente Webers (Anm. 58).

75 Ebd.
} 
delt sind und vor deren Hintergrund sie bewertet werden müssen. ${ }^{76}$ Als „,Dienst an der Wissenschaft ${ }^{677}$ ist darum diese Bereitstellung der Kontexte, auf die Forscherinnen bei der Benutzung der edierten Briefe und darüber hinaus generell für ihre Forschungen zurückgreifen können, ${ }^{78} \mathrm{zu}$ bewerten und auch zu honorieren. Dokumentation, Erschließung, Kommentierung im engeren Sinn, bestmögliche Vernetzung und Bereitstellung verschiedener Nutzungsfunktionalitäten gehören zu einer zeitgemäßen digitalen historisch-kritischen Edition. Sie haben unterschiedliche Funktionen für das Verständnis der edierten Texte und dürfen darum nicht gegeneinander ausgespielt werden. Eine historisch-kritische Edition muss demnach - auch durch eine sorgfältige Kommentierung - die Voraussetzung für unterschiedliche Zugänge zum edierten Korpus schaffen. ${ }^{79}$ Sie kann sich - wohl im Unterschied zu anderen Formen digitaler Editionen - nicht damit begnügen, als „Fluchtpunkt der digitalen (Brief-)Edition [...] die Summe der vielfältig durchsuchbaren und weitestgehend normierten Metadaten“ zu definieren. ${ }^{80}$ Gewiss spiegeln sich ,[g]egenwärtige Interessen, Kompetenzen und auch finanzielle Mittel [...] in solchen Niveauunterschieden" digitaler Editionen wider, und diese Varianz ist durchaus als positiv zu bewerten ${ }^{81}$ - aber nur dann, wenn sie Ausdruck einer noch nicht abgeschlossenen Entwicklungsphase ist, in der Editionen mit unterschiedlichen Ansprüchen und Traditionen nebeneinander bestehen (dürfen). Wenn die Digitale Schlegel-Edition „durch best practice am gegenwärtigen Standardisierungsprozess für digitale Editionen mitwirken“" möchte, so ist zu hoffen, dass die Äußerung auf Textwiedergabe, semantische Erschließung, Erschließung von Entitäten, Navigation und Präsentation abzielt und nicht den Förderinstitutionen den Rotstift zur generellen Streichung von Editionskommentaren in die Hände spielt. ${ }^{82}$ Dies käme der Abkehr von einem Standard gleich, der sich in den letzten Jahrzehnten gerade für gedruckte Briefeditionen mit guten Gründen durchgesetzt hat.

76 Vgl. auch Christian von Zimmermann: Jeremias Gotthelf und der Neue Berner-Kalender - Überlegungen zu einem Kommentar in der historisch-kritischen Edition. In: Akten des XI. Internationalen Germanistenkongresses Paris 2005 „Germanistik im Konflikt der Kulturen“. Hrsg. von Jean-Marie Valentin. Bd. 5: Kulturwissenschaft vs. Philologie? Bern u. a. 2007 (Jahrbuch für Internationale Germanistik. Reihe A. Kongressberichte. 81), S. 293-299, hier S. 296 und S. 298.

77 Leuschner 2013 (Anm. 11), S. 227.

78 Siehe Woesler 1998 (Anm. 22), S. 79f.; Leuschner 1993 (Anm. 29), S. 187.

79 Vgl. von Zimmermann 2014 (Anm. 21), S. 236f.

80 Strobel 2014 (Anm. 19), S. 153.

81 Ebd.

82 Digitale Edition der Korrespondenz August Wilhelm Schlegels (Anm. 39; http://august-wilhelm-schlegel. de/briefedigital/info/project). - Kritisch zur Förderpolitik siehe Andreas Arndt: Schleiermacher edieren. In: editio 30, 2016, S. 157-167, hier S. 166: „Dass dies rein wissenschaftlich gesehen nicht vertretbar ist, weil eine zuverlässige Edition ohne solche Bemühungen [interpretatorische] gar nicht möglich ist, ist schon oft betont worden." 